\title{
Unclear Mechanism of Cardiopulmonary Arrest Following Cervical Epidural Steroid Injection
}

\section{TO THE EDITOR}

We read with much interest the case report titled 'Cardiopulmonary Arrest Following Cervical Epidural Steroid Injection' by Stauber et al (1) in the March/April 2012 issue. The case report highlighted a cardiopulmonary arrest which occurred within 5 seconds after the injection of epidural steroid in $1 \%$ lidocaine. The quoted amount of betamethasone was $12 \mathrm{mg}$ in a total volume of $4 \mathrm{~mL}$. Assuming a standard concentration of $6 \mathrm{mg}$ per milliliter, this would indicate two milliliters of $1 \%$ lidocaine were injected into the epidural space. The authors maintain that the cardiopulmonary arrest was caused from the blockade of sympathetic cardiac accelerator fibers immediately following the injection at C6-C7. It was also postulated that the pneumocephalus that was later found on CT scan was the result of a similar "pump" mechanism proposed by the Imanishi article (2) which describes the finding of air in intracranial veins after CPR.

A more likely cause that would account for the case details is intrathecal administration of lidocaine as the culprit for subsequent cardiac arrest. The fact that the pneumocephalus found on CT was subarachnoid and that loss of resistance to air was the method used to locate the epidural space leads me to believe that it is more probable that the air entry site was also subarachnoid. Imanishi's article explains the rare finding of air in intracranial veins after external cardiac massage but does not adequately explain the finding of subarachnoid air.

It would be instructive to see the fluoroscopy images after the $2 \mathrm{~mL}$ of iodinated contrast agent had been injected. Depending on the patient's body habitus and the fluoroscopy views used, it is certainly possible that there was either an inadvertent rent in the dura matter or even intrathecal spread of contrast which was not recognized. These findings would explain not only the pneumocephalus but also the rapidity of which cardiovascular collapse occurred after injection.)

This case report also highlights one of the rare but potential dangers for even "routine" procedures. Although it has not been our standard practice to start a peripheral intravenous line in all patients undergoing cervical procedures, our practice may change in the future.

\author{
Stephen Markewich, MD \\ Department of Anesthesia and Pain \\ University of North Carolina \\ Chapel Hill, NC \\ E-mail: smarkewich@primecare.org \\ Manoj Wunnava MD \\ Department of Anesthesia and Pain \\ University of North Carolina \\ Chapel Hill, NC \\ E-mail: mwunnava@aims.unc.edu
}

\section{References}

1. Stauber B, Ma L, Nazari R. Cardiopulmonary arrest following cervical epidural injection. Pain Physician 2012; 15:147-152.
Imanishi M, Nishimura A, Tabuse $H$, Miyamoto S, Sakaki T, Iwasaki S. Intracranial gas on CT after cardiopulmo- nary resuscitation: 4 cases. Neuroradiology 1998; 40:154-157

\section{Response:}

In response to "Unclear mechanism of Cardiopulmonary Arrest Following Cervical Epidural Steroid Injection," the author brings to light a very plausible mechanism to explain the aforementioned cardiac arrest. While we had considered the mechanism of an in- trathecal injection (and in fact touched on this in terms of phrenic nerve involvement in our discussion), we ultimately shied away from this conclusion. The most compelling reason was the almost immediate onset of the cardiopulmonary arrest following the injection. To the 
best of our knowledge, such an injection, even with $2 \mathrm{ml}$ of lidocaine, would not cause such a profound reaction with 5 seconds. However we believe this mechanism to certainly be plausible and aside from the time factor, have no reason to dispute it.

We agree that the fluoroscopy images would have indeed been helpful. Unfortunately they are not available to us for review. The authors of the manuscript took over care of this patient after she presented to the emergency department, while the patient's original procedure occurred at an outside surgical center. We thank the author for the interest in our manuscript.
Bradley Stauber, DO

Touro University

College of

Osteopathic Medicine

Vallejo, CA.

Reza Nazari, MD, FACC

Medical Director

Chest Pain Center

Emanuel Medical Center

Turlock, CA.

825 Delbon Ave

Turlock, CA 95382

Email: Reza.Nazari@emanuelmed.org 